\title{
A proposed classification system of central airway stenosis
}

\author{
L. Freitag*, A. Ernst" ${ }^{\#}$, M. Unger ${ }^{\dagger}$, K. Kovitz ${ }^{+}$and C.H. Marquette ${ }^{\S}$
}

\begin{abstract}
Tracheobronchial stenosis, a serious problem in adults and children, has multiple causes and has been treated in many ways. While developing an international multicentre study to evaluate efficacy of airway stents, it was realised that no adequate description of central airway stenosis regarding type, location and degree has been published. Thus, comparing results of different treatment modalities in different centres has been difficult due to a lack of uniformity of classification. Reports are typically descriptive and precise classification schemes have not adequately addressed either for the trachea or the main bronchi.
\end{abstract}

A standardised classification scheme was proposed with descriptive images and diagrams for rapid and uniform classification of central airway stenosis. The present authors' system divides stenosis into structural and dynamic types and further classifies the disease by degree of stenosis, location and transition zone.

Multiple sites can be described and each is transformed into a simple numerical scoring system prompted by a diagram, which can be easily captured for subsequent uniform analysis across sites.

A pilot validation of the system, with 18 pulmonologists of varying training background, showed strong precision and agreement between observers. Such a system will enhance the ability to study the effectiveness of treatment modalities for central airway stenosis.

\section{KEYWORDS: Airway, airway narrowing mechanisms, bronchofibrescopy, stenting}

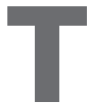

racheobronchial stenosis is a serious problem that affects adults and children. It has multiple causes and has been treated in many ways. Interventional endoscopic procedures compete with surgical approaches and various new treatment modalities are currently being tested for efficacy and safety. While developing an international multicentre study to evaluate the efficacy of airway stents, the present authors realised that no adequate description of central airway stenosis regarding type, location and degree has been published. Thus, comparing results of different treatment modalities in different centres has been difficult due to a lack of uniformity of classification. Reports are typically descriptive and precise classification schemes have not adequately addressed either for the trachea or the main bronchi. A standardised classification scheme was proposed with descriptive images and diagrams for rapid and uniform classification of central airway stenosis.

\section{EARLIER SYSTEMS}

Laryngotracheal stenosis (LTS) in paediatric patients was first classified by COTTON [1]. The classification is based on the cross-sectional area of the stenosis and is divided into four grades.
Location and length are noted but these do not affect the grade of stenosis. This classification does not apply to the lower trachea or bronchi. It was designed for a group of patients who typically had scarring after prolonged intubation. GRUNDFAST et al. [2] retrospectively looked at a paediatric population for factors predictive of outcome in patients treated for subglottic stenosis and opted for a more descriptive form of classification. Like COTTON [1], GRUNDFAST et al. [2] addressed the airway opening by measuring its diameter at the narrowest point. The measurement of length and four types of consistency, i.e. soft, hard, cartilaginous and mixed, was added to this.

More recently, MCCAFFREY [3] retrospectively reviewed the treatment of 72 cases of LTS. Although diameter and length were factors, the predominant predictor of outcome was location. Locations were confined to the glottis, subglottic area and upper trachea. Four stages were defined as follows. 1) Stage 1 in the subglottis or trachea, $<1 \mathrm{~cm}$ in length. 2) Stage 2 in the subglottis, $>1 \mathrm{~cm}$ in length. 3) Stage 3 in the subglottis and upper trachea. 4) Stage 4 in the glottis with vocal cord fixation and paralysis. Outcomes were
AFFILIATIONS

*Lungenklinik Hemer, Hemer, Germany.

${ }^{\#}$ Beth Israel Deaconess Medical Center, Boston, MA

'Fox Chase Cancer Center Philadelphia, PA, and,

+Tulane University Medical Center, New Orleans, LA, USA.

${ }^{\S}$ Hopital Albert Calmette, CHRU de Lille, Lille, France.

CORRESPONDENCE

L. Freitag

Lungenklinik Hemer

58675 Hemer

Germany

Fax: 492372914945

E-mail: freitag-hemer@t-online.de

Received:

December 232004

Accepted after revision:

March 212007

STATEMENT OF INTEREST

None declared.

European Respiratory Journal Print ISSN 0903-1936 Online ISSN 1399-3003 
worse with each advancing stage. GRILLO et al. [4] reviewed their experiences with LTS confined to the upper trachea and subglottic larynx. The stenoses were descriptively characterised and three types were identified. These were in the upper trachea, the subglottis (with a space below the vocal cords for a possible anastomosis) and the subglottis, without an adequate space for an anastomosis.

ANAND et al. [5] also looked at surgical approaches. Specifically, while reviewing the treatment of tracheal stenosis, mostly postintubation, stenosis was characterised based on severity, location, length and number of stenoses. For severity they borrowed from the classification by COTTON [1], but divided it into three grades: mild $(<70 \%)$; moderate $(71-90 \%)$; and severe $(>90 \%)$. Locations were defined as glottic, cervical or thoracic. Lengths were divided into $<1 \mathrm{~cm}, 1-3 \mathrm{~cm}$ and $>3 \mathrm{~cm}$. Multiple areas of stenosis were found in some patients. More recently, MYER et al. [6] determined the degree of tracheal stenosis by using standard endotracheal tubes as guides and observing how they pass through the narrowest point. By comparing with age-appropriate endotracheal tube sizes one can classify four grades of stenosis: grade I ( $\leqslant 50 \%)$; grade II (51-70\%); grade III $(>70 \%)$; and grade IV (complete obstruction).

Distributions with a coefficient of variation $(\mathrm{CV})<100 \%$ are considered high-precision and low-variance, while those with a CV $>100 \%$ are considered low-precision and high-variance.

The major focus of these previous studies [1-6] has been a retrospective attempt to discern outcome of various surgical procedures for the treatment of LTS. All attempts at classifications have concentrated on the upper airway with none extending beyond the trachea. While these descriptive classifications are useful, they do not allow for easily reproducible and analysable comparisons of results between centres. Newer types of interventional bronchoscopy, including laser, dilatation and stent placement, have been discussed in various publications. Their efficacy and place value as an alternative to surgical approaches can only be defined if outcome descriptors are comparable. The lack of a universal classification system limits a true comparison of approaches with respect to outcomes.

\section{PROPOSED CLASSIFICATION OF TRACHEOBRONCHIAL STENOSIS}

A simple bronchoscopic scheme was developed and tested for the classification of tracheobronchial stenosis that will allow different centres to prospectively compare results and analyse outcome over a wide range of interventions. This classification system is proposed in the hope that the system will be

\begin{tabular}{lcc} 
TABLE 1 & Stenosis groupings & \\
Stenosis & Type & Character \\
\hline \multirow{2}{*}{ Structural } & 1 & Exophytic/intraluminal \\
& 2 & Extrinsic \\
& 3 & Distortion \\
Dynamic or functional & 4 & Scar/stricture \\
& 1 & Damaged cartilage/malacia \\
& 2 & Floppy membrane \\
\hline
\end{tabular}

\section{TABLE 2 Numerical assignment of degree}

\begin{tabular}{lc} 
Code & Degree \% \\
\hline $\mathbf{0}$ & No stenosis \\
$\mathbf{1}$ & $<25^{\#}$ \\
$\mathbf{2}$ & $26-50^{\#}$ \\
$\mathbf{3}$ & $51-75^{\#}$ \\
$\mathbf{4}$ & $76-90^{\#}$ \\
$\mathbf{5}$ & $90-$ complete obstruction \\
\hline \#: decrease in cross-sectional area. &
\end{tabular}

accepted by other centres or, at least, stimulate further discussion in the European Respiratory Journal.

A classification method has been proposed that can be used simply and reproducibly between different centres for the characterisation of tracheobronchial stenosis. Such a system will lend itself to placing stenoses into a specific and finite number of categories that can be more easily collated. This classification system should then allow for a quantitative analysis of outcome. Diagnostic and clinical examples were included with a suggested charting device to ease implementation. Specialists from six major institutions in Europe and the USA have agreed to this system.

\section{Types of stenosis}

There are two major groups of stenosis: structural and dynamic or functional (table 1).

\section{Structural stenosis}

The structural group has four major types described as follows. 1) Type 1 stenosis includes all types of exophytic intraluminal malignant or benign tumours and granulation tissue. 2) Type 2 stenosis is due to extrinsic compression, such as from enlarged lymph nodes, goitre, large vessels or other mediastinal structures, including nonpulmonary tumours. 3) Type 3 stenosis is due to distortion, kinking, bending or buckling. These may be complications seen after surgical interventions, such as sleeve resections, transplantation and the like, as well as mediastinal or pleural diseases causing traction of the bronchi. Although the bronchial wall thickness may be normal, off-axis distortion results in oval shaped stenotic segments. 4) Type 4 stenoses are cases of stenosis in which shrinking and scarring are predominant. Post-intubation stenosis, burn injuries and secondary healing after surgery are typical examples.

\section{TABLE 3 Scoring system according to location}

\section{Location}

Upper third of the trachea Middle third of the trachea Lower third of the trachea

Right main bronchus Left main bronchus 
a)

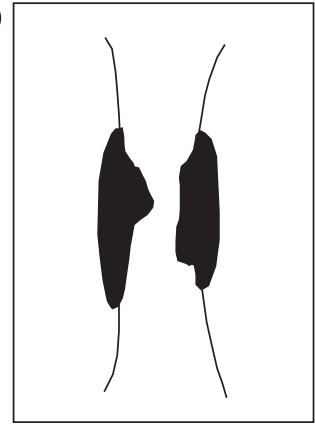

e)

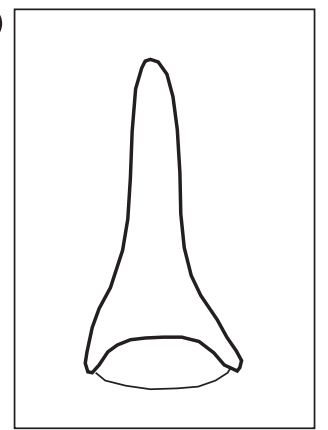

b)

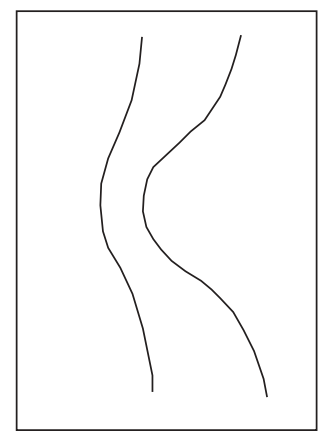

f)

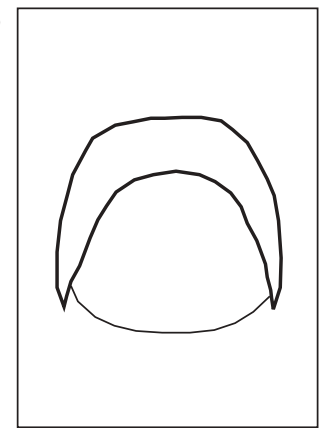

c)

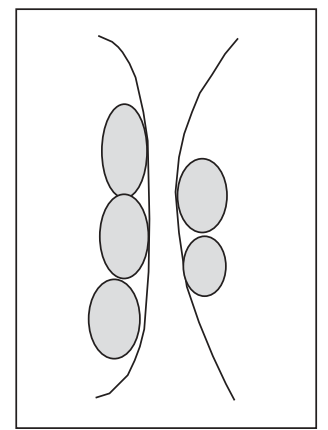

g)

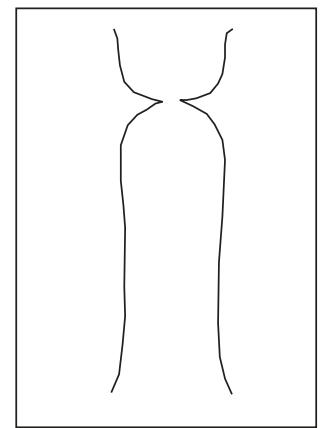

d)

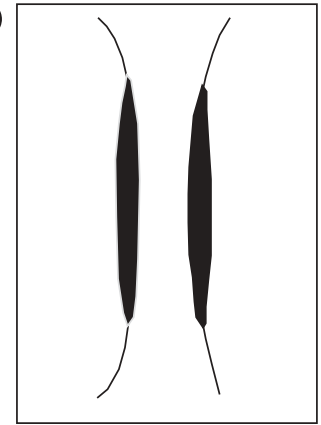

h)

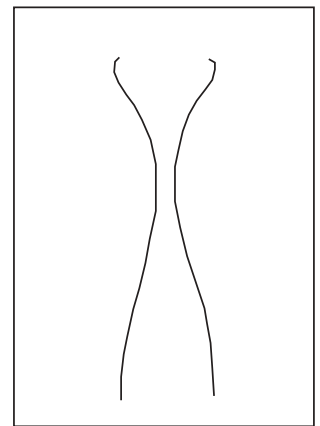

FIGURE 1. Schematic representations of the basic types of stenosis and the transition or abruptness of change between the normal lumen and the most narrowed part. a) Intraluminar tumour or granulation; b) distortion or buckling; c) extrinsic compression; d) scar stricture; e) scabbard trachea; f) floppy membrane; g) abrupt transition (web stenosis); h) tapered transition (hour glass stenosis).

Dynamic (functional) stenosis

Dynamic stenoses are malacic conditions that vary with the respiratory cycle. They include two different types. Type 1 is the triangular (tent)-shaped benign stenosis in which the cartilage is damaged. This sabre sheath (scabbard) trachea should be distinguished from the more common type 2, which
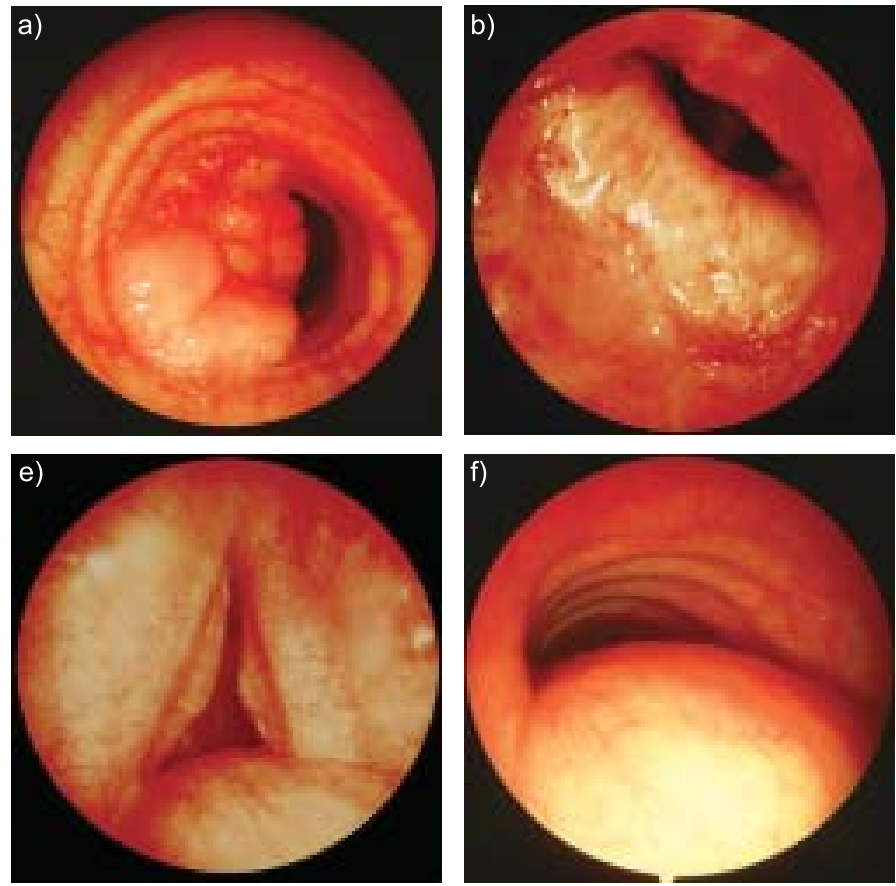
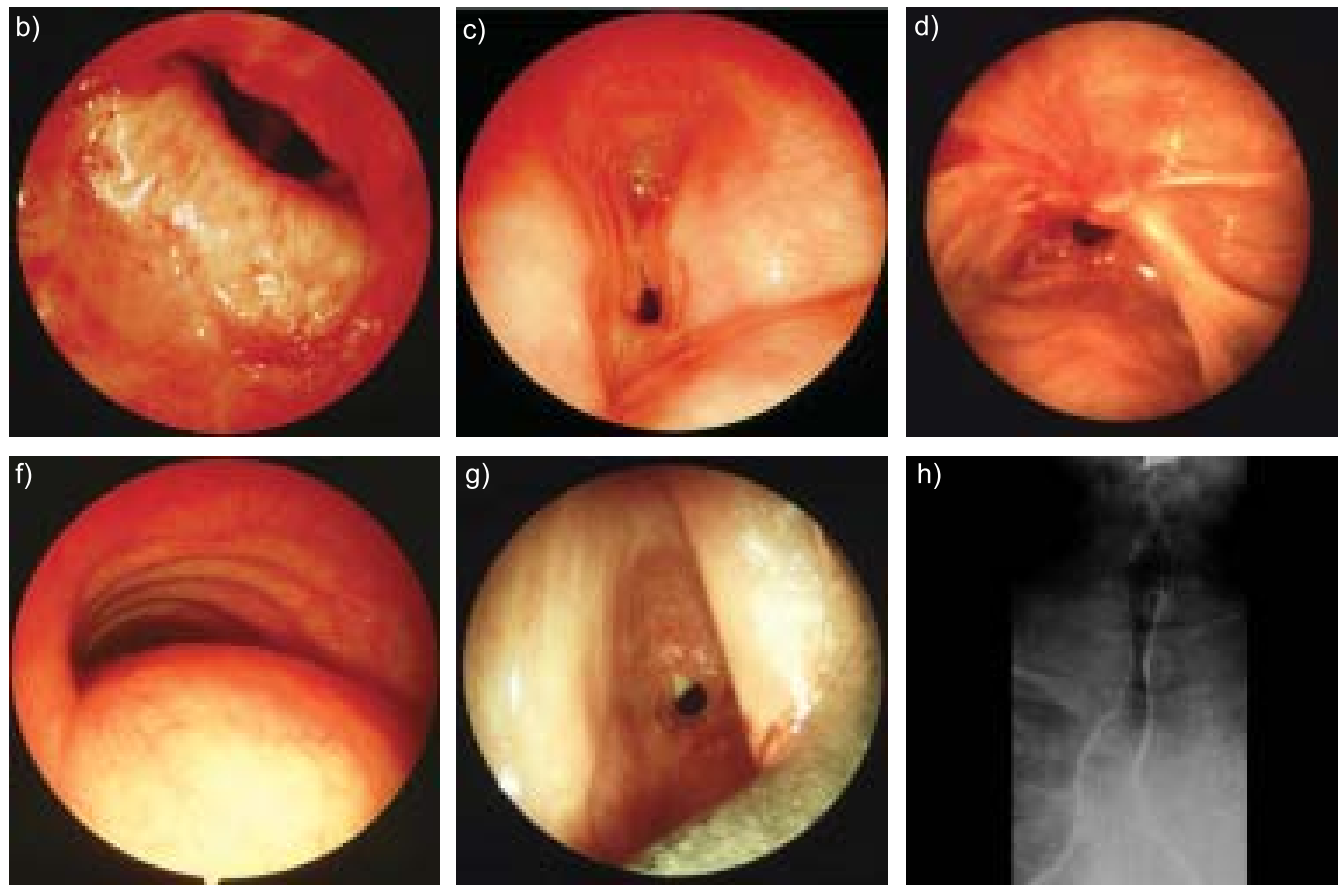

FIGURE 2. Clinical examples of the degrees of stenosis. a) Intraluminar tumour or granulation; b) distortion or buckling; c) extrinsic compression; d) scar stricture: e) scabbard trachea; f) floppy membrane; g) abrupt transition (web stenosis); h) tapered transition (hour glass stenosis). 

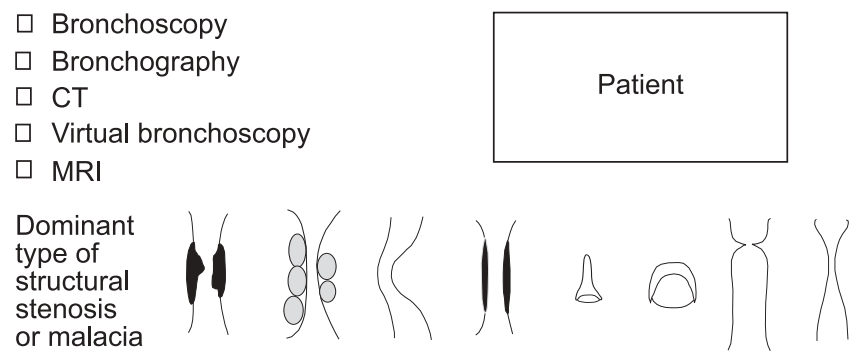

Type of

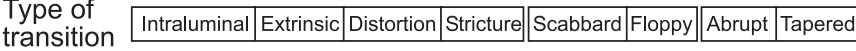

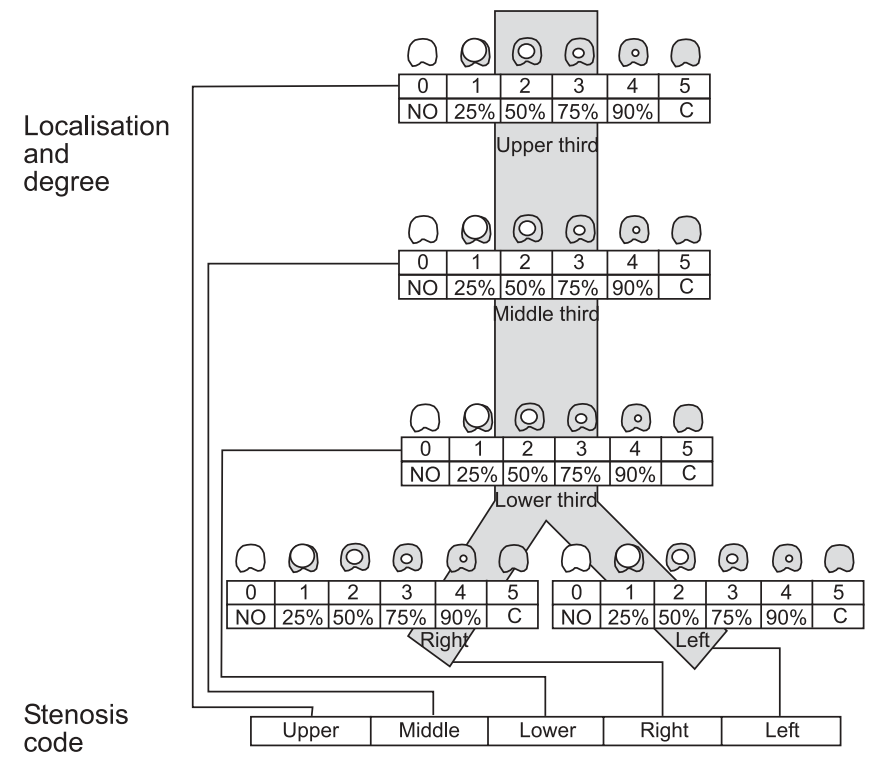

FIGURE 3. A worksheet marking the location, degree and type of stenosis. CT: computed tomography; MRI: magnetic resonance imaging; C: complete.

is often seen in emphysema patients, where there is an inward bulging of the floppy posterior membrane.

\section{Degree of stenosis}

The degree of stenosis is assigned a numerical code that can be applied to any site (table 2). Code 0 is assigned to nonappreciable stenosis and codes 1, 2, 3 and 4 are assigned, respectively, to an $\sim 25,50,75$ and $90 \%$ decrease in cross-sectional area. Code 5 is assigned to complete obstruction. For dynamic stenoses, the condition during forced exhalation is considered. Operator judgment is used to assign the closest appropriate degree.

\section{Location}

The method proposed in the current study defines five locations within the central airways: 1) location I, upper third of the trachea; 2) location II, middle third of the trachea; 3) location III, lower third of the trachea; 4) location IV, right main bronchus; 5) location $\mathrm{V}$, left main bronchus (table 3 ). In the resulting scoring system, which lends itself readily to a computerised system, the stenosis location will be at the appropriate position of the numerical scoring. That is, each stenosis will have five digits assigned to it. The degree of stenosis is reported in the first through to the fifth numerical position representing the first through to the fifth location
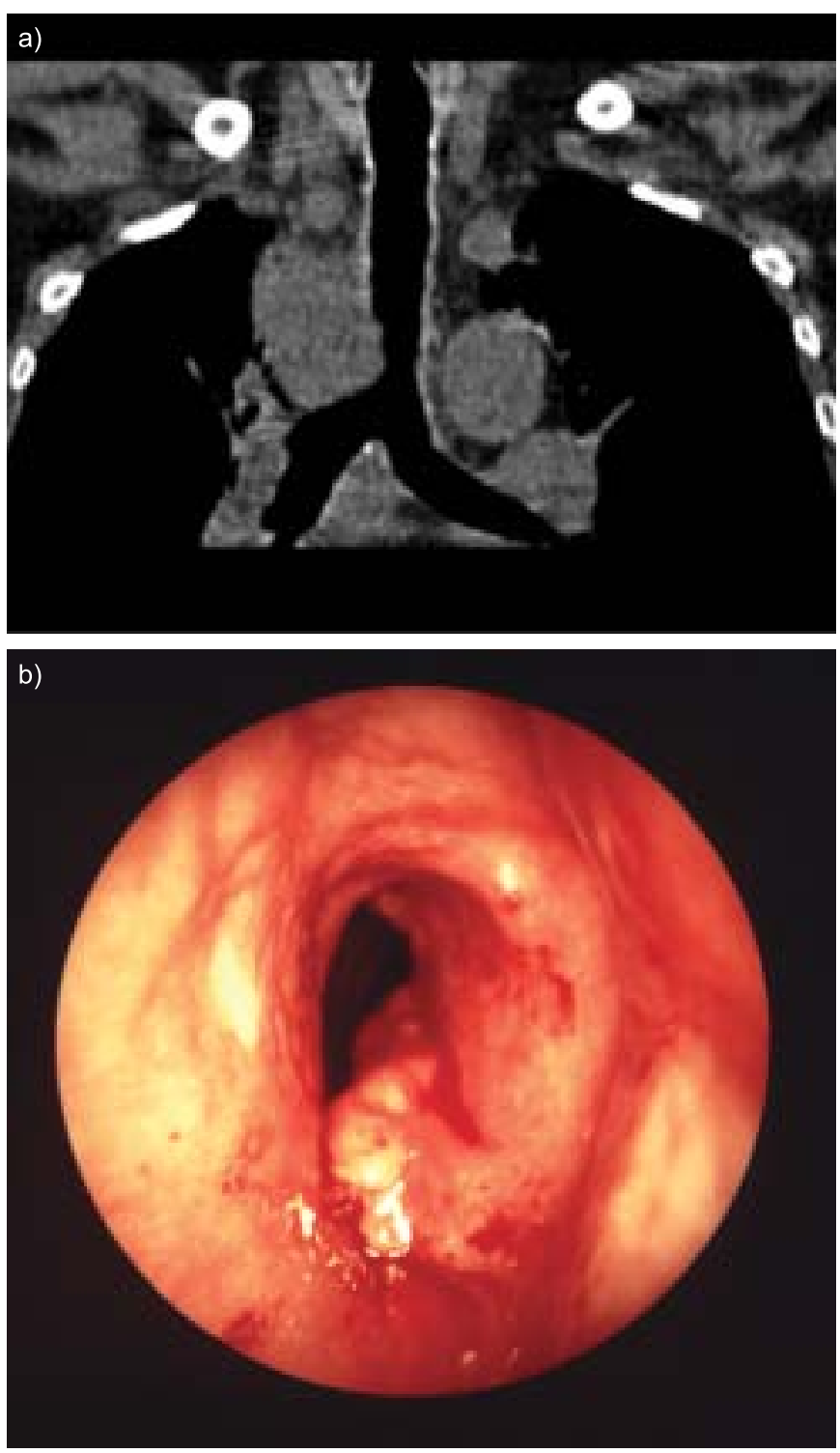

FIGURE 4. Example of an airway stenosis classification.

number. Thus, for instance, $50 \%$ stenosis in the lower trachea will be represented by the computerised scoring system in the following way: 00300. In the third position (location III) the number 3 represents a $50 \%$ reduction in the lumen, while the rest of the evaluable tracheobronchial tree is within normal limits.

\section{Transition zone}

For decisions regarding therapeutic approaches, not only the overall length but also the transition or abruptness of stenosis is relevant. The description should include information where there is an abrupt change, such as a web stenosis, or a slow bottleneck-type transition as in a cicatricial post-intubation stenosis.

\section{Charting}

For ease of use, diagrammatic representations of the degrees of stenosis are, and can be, incorporated in a standard report 


\section{TABLE 4 Precision data summary}

\begin{tabular}{lcc} 
Variable & Cases $\mathbf{n}$ & Coefficient of variation \% \\
\hline Upper trachea & 10 & 18 \\
Middle trachea & 10 & 27 \\
Lower trachea & 10 & $\sim 0$ \\
Right stem & 10 & $\sim 0$ \\
Left stem & 10 & $\sim 0$ \\
\hline
\end{tabular}

form (figs. 1 and 2). These visual guides can be used to help assign numbers. For practical reasons, a graphical file worksheet (fig. 3) has been created that can be easily adapted by individual institutions. This worksheet can be downloaded from the present authors' website. The description was originally based on endoscopic findings but can be correlated with radiological findings, including bronchography, computed tomography, virtual bronchoscopy or magnetic resonance imaging (fig. 4). Other techniques of measurement, such as acoustic reflection [7] or the use of designated bronchoscopic equipment $[2,8]$, might be used. Correlations between this descriptive code and functional data $[9,10]$ are currently being collected and will be presented in a follow-up paper.

\section{Validation}

To assess feasibility of the proposed classification system, 18 participants in an Interventional Pulmonology course (Lille, France) were asked to independently assess 10 cases using this system. After a short instruction session, participants were able to review a video of the bronchoscopy, as well as a description of the findings in a conventional endoscopy report. The group of participants consisted of four fellows and 14 pulmonary specialists. The time spent in clinical practice ranged 1-20 yrs.

The coefficient of variation (CV) was used to measure variability and precision, while the Pearson's rho correlation coefficient was used to measure agreement. Distributions with $\mathrm{CV}<100 \%$ are considered high precision and low variance, while those with $\mathrm{CV}>100 \%$ are considered low precision and high variance. Correlations of $100 \%$ indicate perfect agreement between raters, while correlations of $0 \%$ indicate complete nonagreement.

The precision and agreement data are presented in tables 4 and 5 , respectively. There was strong precision and agreement

\section{TABLE 5 Agreement between raters}

\begin{tabular}{lcc} 
Variable & Cases $\mathbf{n}$ & Summary correlation rho \% \\
\hline Upper trachea & 10 & 86 \\
Middle trachea & 10 & 67 \\
Lower trachea & 10 & 97 \\
Right stem & 10 & 99 \\
Left stem & 10 & 99 \\
\hline
\end{tabular}

Correlations of $100 \%$ indicate perfect agreement between raters, while correlations of $0 \%$ indicate complete nonagreement. between raters, with the strongest precision and agreement for abnormalities in the lower trachea, as well as right and left mainstem. In the following discussion it transpired that most of the discrepancies could be explained by the fact that some participants marked the degree of stenosis during inspiration, while others used the minimal cross-sectional area at the end of expiration. For future studies it was proposed to rate the highest degree of stenosis during normal breathing, which in most cases is at the end of expiration.

\section{CONCLUSION}

In essence, a simple system was proposed to distinguish between the different types of airway stenosis. It can be used prospectively to numerically classify stenosis of the tracheobronchial tree. It allows for the assignment to multiple points within the large airways of numerical representations of the degree and sites of stenosis. The system, therefore, accommodates the full range of patients; from those with a single area of simple stenosis to those with multiple complex stenoses. The numerical assignment of grades that are easily gleaned from a diagnostic template allows for both rapid classification and subsequent numerical analysis. The pilot validation trial confirms that the system is easy to use and precise.

Several shortcomings need to be addressed. Precision in some areas is not as high as in others and thus some improvement is required. Additionally, the current system does not allow for independent abnormalities in one patient to be scored on one sheet. These issues are areas of active research for improvement and a larger trial is recommended to assess the validity of the present results.

Additionally, possible extensions or improvements of the system could include the larynx and the lobar bronchi. Biomechanical measurements could be included to distinguish between soft and rigid stenosis. However, although more precise, these extensions would complicate the use of the system and it is believed the proposed classification system is feasible for most purposes.

The current authors hope that the system described in the present study will allow for a more uniform and quantitative prospective approach to the analysis of the outcome of the myriad approaches taken in the treatment of tracheobronchial stenosis.

\section{ACKNOWLEDGEMENTS}

The authors gratefully acknowledge the assistance of M. Secic with the statistical analysis.

\section{REFERENCES}

1 Cotton RT. Pediatric laryngotracheal stenosis. J Pediatr Surg 1984; 19: 699-704.

2 Grundfast KM, Morris MS, Bernsley C. Subglottic stenosis: retrospective analysis and proposal for standard reporting system. Ann Otol Rhinol Laryngol 1987; 96: 101-105.

3 McCaffrey TV. Classification of laryngotracheal stenosis. Laryngoscope 1992; 102: 1335-1340.

4 Grillo HC, Mark EJ, Mathisen DJ, Wain JC. Idiopathic laryngotracheal stenosis and its management. Ann Thorac Surg 1993; 56: 80-87. 
5 Anand VK, Alemar G, Warren ET. Surgical considerations in tracheal stenosis. Laryngoscope 1992; 102: 237-243.

6 Myer CM 3rd, O'Connor DM, Cotton RT. Proposed grading system for subglottic stenosis based on endotracheal tube sizes. Ann Otol Rhinol Laryngol 1994; 103: 319-323.

7 Hoffstein V, Zamel N. Tracheal stenosis measured by the acoustic reflection technique. Am Rev Respir Dis 1984; 130: 472-475.

8 Kleinsasser N, Kronsdorf D, Merkenschlager A, et al. Endoskopische, dreidimensionale Vermessung von
Neubildungen und Stenosen des Larynx und der Trachea [Endoscopic, 3-dimensional measurement of neoplasms and stenoses of the larynx and trachea]. Laryngorhinootologie 1994; 73: 428-431.

9 Miller RD, Hyatt RE. Evaluation of obstructing lesions of the trachea and larynx by flow-volume loops. Am Rev Respir Dis 1973; 108: 475-481.

10 Gamsu G, Borson DB, Webb WR, Cunningham JH. Structure and function in tracheal stenosis. Am Rev Respir Dis 1980; 121: 519-531. 